Institute, Tunisia). It included all patients withbreastlesionreferred to our unit. Smearswerefixed and stained by May GrünwaldGiemsa .Cytologyresultswerecategorizedaccording to the NCI by twocytopathologistsindependently.

Methodology The presentstudywascarried out over a 1-year period (January 2017-December 2017) and conductedat the cytology unit of PathologyDepartment (Salah Azaiez Institute, Tunisia). It included all patients withbreastlesionreferred to our unit. Smearswerefixed and stained by May GrünwaldGiemsa . Cytologyresultswerecategorizedaccording to the NCI by twocytopathologistsindependently.

Result(s)* FNAC interpretivecategorizationwas as follows : 188 (31,3\%)inadequate (C1), 378 (63\%) benign (C2), 1 (0,16\%) atypical, probablybenign (C3), $31(5,14 \%)$ suspicious of malignancy (C4) and $2(0,4 \%)$ malignant (C5). The histopathologicalexamination of category C3 showed a benignlesion. Histopathologicalexamination of category C4 wasassessedonly for 20 patients. $14 / 20$ cases showedbenignlesions, in one case an in-situ carcinomawasdiagnosed and 5/20 cases showedmalignancy.The positive predictive value of $\mathrm{C} 4$ category in the diagnosis of malignancywas 30\%.

Conclusion* FNA is a safe and cost-effective technique for the diagnosis of breastlesions, especiallywhencorrelatedwithclinical and imagingstudies. It has achievedgreat importance in diagnosis and management of palpable breastlesions. However, we must beaware of possibility of "false positive" and "false negative" resultsespecially for categories C3 and C4.

\section{IMPACT OF QUALITY CONTROL PROCEDURE IN THE MANAGEMENT OF BREAST BIOPSY}

${ }^{1} S$ Sakhri, ${ }^{2} \mathrm{~S}$ Kammoun, ${ }^{2} \mathrm{~T}$ Karima, ${ }^{2} \mathrm{~K}$ Hamza, ${ }^{1} \mathrm{M}$ Bouhani*, ${ }^{2} \mathrm{~A}$ Goucha, ${ }^{2} \mathrm{~B}$ Bettaib, ${ }^{2} \mathrm{M}$ Driss. ${ }^{1}$ Institut Salah Azaiz, surgical departement, Tunis, Tunisia; ${ }^{2}$ Institut Salah Azaiz, anatomopathology, Tunis, Tunisia

\subsection{6/ijgc-2021-ESG0.536}

Introduction/Background* Breast cancer is the first women cancer. For adequate treatment we should have a relevant diagnosis based on morphology and immunohistochemistry (IHC). In our national institute of oncology the deadlines of pathology report are very long because of the significant number of samples received from different department. We have established a specific protocol to manage breast biopsy to speed up the histological report. We would represent in this paper the evaluation of the implantation of this support protocol in our lab.

Methodology In the new procedure of our lab the breast biopsy was separately treated from other routine samples. They was treated in cassette of different color. Two technicians was specially dedicate to manage all the technical steps. In our laboratory we received both chirurgical and radiological biopsy. Both go under the same technical protocol. They are fixed with formalin then they goes through the preanalytical procedures the same day. To evaluate the impact of the implement of this procedure, we compare the report output deadline of biopsy received between January and February 2018 with those received the same period in 2020 after the implementation of the protocol.

Result(s)* In 2018, the deadlines of issuing the final report were on average 21.5 day while in 2020 after implementation of the protocol, the deadlines issuing passed to 11.75 day. The deadlines stay long because of many others issues like long absence of patients especially for socio-economic reasons.
For us in the laboratory we can guarantee a primary report based on morphological features within 2 days after receiving the biopsy. The final report with IHC report can be ready in maximum 6 days after receiving the sample. In fact it depends on the rhythm of the IHC technique which is programmed minimum twice per week.

Conclusion* Pathology report is the key of all therapeutic issues in breast cancer. For a best supported therapy for our patients we accelerate the procedure of biopsy management while keeping a good quality of study. Our ultimate goal is to provide the most specific diagnosis as soon as possible to procure the best therapy for patients.

\section{UPDATE ON TRIPLE-NEGATIVE BREAST CANCER: THE GOOD PROGNOSIS}

${ }^{1} S$ Sakhri, ${ }^{2} \mathrm{~K}$ Hamza, ${ }^{2} \mathrm{~S}$ Kamoun, ${ }^{2} \mathrm{Y}$ Houcine, ${ }^{2} \mathrm{~T}$ Karima, ${ }^{1} \mathrm{M}$ Bouhani* ${ }^{2}{ }^{2} \mathrm{~B}$ Bettaib ${ }^{2} \mathrm{M}$ Driss. 'Institut Salah Azaiz, surgical departement, Tunis, Tunisia; ${ }^{2}$ Institut Salah Azaiz, anatomopathology, Tunis, Tunisia

\subsection{6/ijgc-2021-ESG0.537}

Introduction/Background* Triple-negative breast cancer(TNBC) represent a consistent subgroup of breast cancers with heterogeneous clinical presentation, clinical behavior, histology, and response to therapy. Awareness of TNBC was recently increased by the discovery of the intrinsic molecular subtypes in breast cancers with gene expression profiling experiments. At least five molecular categories have been repeatedly identified in breast cancer, each with prognostic significance.

Methodology We retrospectively reported 18 cases of tripe negative cancer treated at salah azaiez institute Tunisia between 2016 and 2021.including 3 cases of CAK (adenoid cystic carcinoma), 10cases of CI-TNS-CM (medullary carcinoma) et 5 cases de CA (apocrine carcinoma).

Result(s)* The average age was 68 years. The mean tumor size was $37 \mathrm{~mm}$. In $15 / 18$ cases the tumors were grade III SBR modified. Lymphatic emboli were observed in 4 cases/18. The Ki67 was on average 57\%. Metastatic lymph nodes were identified in 4 cases out of 14 having had axillary dissection. Conclusion* The prognostic value of classical pathological variables such as tumor grade, lymph node status, and tumor size, could be impaired in TNBC. Indeed, most TNBC are high grade, and first generation prognostic molecular assays, which are driven mainly by proliferation, did not show prognostic value in TNBC. In contrast, Ki67 stainings in TNBC have been suggested to carry prognostic information in some reports but not in others.8Small (cT1a/b) node-negative TNBC are potentially aggressive as well. Despite the abovementioned issues, the Nottingham Prognostic Index (NPI) has been reported to be useful in TNBC.

\section{THE MOLECULAR PROFILE OF CUTANEOUS METASTASIS FROM BREAST CANCER}

${ }^{1} \mathrm{~S}$ Sakhri, ${ }^{2} \mathrm{~S}$ Kammoun, ${ }^{2} \mathrm{~K}$ Hamza, ${ }^{2} \mathrm{Y}$ Houcine, ${ }^{1} \mathrm{M}$ Bouhani* ${ }^{2}{ }^{2}$ Bettaieb, ${ }^{2} \mathrm{M}$ Driss ${ }^{1}$ Institut Salah Azaiz, surgical departement, Tunis, Tunisia; ${ }^{2}$ Institut Salah Azaiz, anatomopathology, Tunis, Tunisia

\subsection{6/ijgc-2021-ESG0.538}

Introduction/Background* The incidence of breast carcinoma cutaneous manifestation in patients with breast carcinoma is 23.9\%. The most common sites of breast carcinoma cutaneous 
manifestation are the chest wall and abdomen, but they can occur at the extremities and in the head/neck region. Due the high incidence of breast carcinoma, these cutaneous manifestations are the most common metastases seen by dermatologists. Methodology We retrospectively reported 20 cases of cutaneous metastasis from breast carcinoma diagnosed at salah azaiez institute Tunisia between 2015 and 2021.

Result(s)* there were a total of 20 cases of cutaneous metastasis from breast cancer. The average time interval between diagnosis of breast cancer and cutaneous presentation was 4years. The cutaneous sites of involvement included the head and neck (5), the trunk (10), the extremities (3), and multiple sites (2). The age range was 38-83 <months, and the average survival following diagnosis was 3 years. The treatment consisting on surgical resection and chemotherapy in the majority of cases. Histological examination showed that in 4 cases skin metastasis change molecular profile.

Conclusion* Skin metastasis from breast cancer is frequent . The diagnosis is easy by biopsy or surgical excision. The molecular profile is variable according to the behavior and the aggressiveness of the primary tumor, an in-depth histological study is necessary to dictate the management.

\section{Prevention of gynaecologic cancer}

\section{PREDICTOR FACTORS FOR CONSERVATIVE MANAGEMENT OF CERVICAL INTRAEPITHELIAL NEOPLASIA GRADE 2: CYTOLOGY AND HPV GENOTYPING}

A Salvado, E Miralpeix Rovira*, JM Sole-Sedeno, N Kanjou, S Espuelas, G Mancebo. Hospital del Mar, Barcelona, Spain

\subsection{6/ijgc-2021-ESG0.539}

Introduction/Background* Cervical intraepithelial neoplasia (CIN) grade 2 is classified as high-grade intraepithelial lesion (HSIL) due to its potential for developing cancer. Persistent human papillomavirus (HPV) infection is an established risk factors to develop cervical cancer and its precursor lesions. The management of CIN2 remains under discussion, spontaneous progression and regression rates are around 11$18 \%$ and $50-61 \%$ respectively at 24 months of follow-up. The purpose of this study was to evaluate the role of HPV genotyping and previous cytology result to predict the evolutions of CIN2 managed conservatively.

Methodology A prospective observational study was conducted at Hospital del Mar in Barcelona from January 2012 to May 2017. Women with new diagnosis of CIN2 were invited to undergo conservative management for 24 months. Complete regression, partial regression, persistence, and progression to CIN3 were defined as final outcomes.

Result(s)* 291 of the 300 included patients completed the 24months follow-up. Of them, 214 patients (73.5\%) showed regression; 43 (14.8\%) persistence of CIN2, and $34(11.7 \%)$ progression to CIN3. In multivariable analysis, HPV-16 infection (odds ratio [OR] 1.97 [95\% confidence interval $\{\mathrm{CI}\}$ 1.13-3.43]) and previous cytology (OR 3.46, 95\% CI 1.99-6.02) significantly increased the risk of persistence or progression of CIN2 lesions $(\mathrm{CIN} 2+)$. All HPV-negative lesions regressed $(\mathrm{p}<0.001)$. According to patients' age, no significant differences between age at diagnosis and final diagnosis were found.

Conclusion* The regression rate of CIN2 lesions supports conservative management in selected patients regardless of their age. Patients with CIN2 biopsy and negative HPV test had a high rate of regression and should be offered follow-up without excisional treatment. Women with HPV-16 and HSIL cytology had an increased risk of $\mathrm{CIN} 2+$, their treatment should be individualized, and excisional treatment should be considered.

\section{3 "SEE AND TREAT": AN ADVOCATED PERSPECTIVE FOR PRE INVASIVE LESIONS OF THE CERVIX}

A Gaurav*, D Kapur, K Khoiwal, S Choudhuri, AK Ravi, J Chaturvedi. All India institute of medical sciences, Rishikesh, Obstetrics and Gynaecology, RISHIKESH, India

\subsection{6/ijgc-2021-ESGO.540}

Introduction/Background* Secondary prevention of cervical cancer should remain a key priority for women's health globally for decades to come, especially in developing countries. A

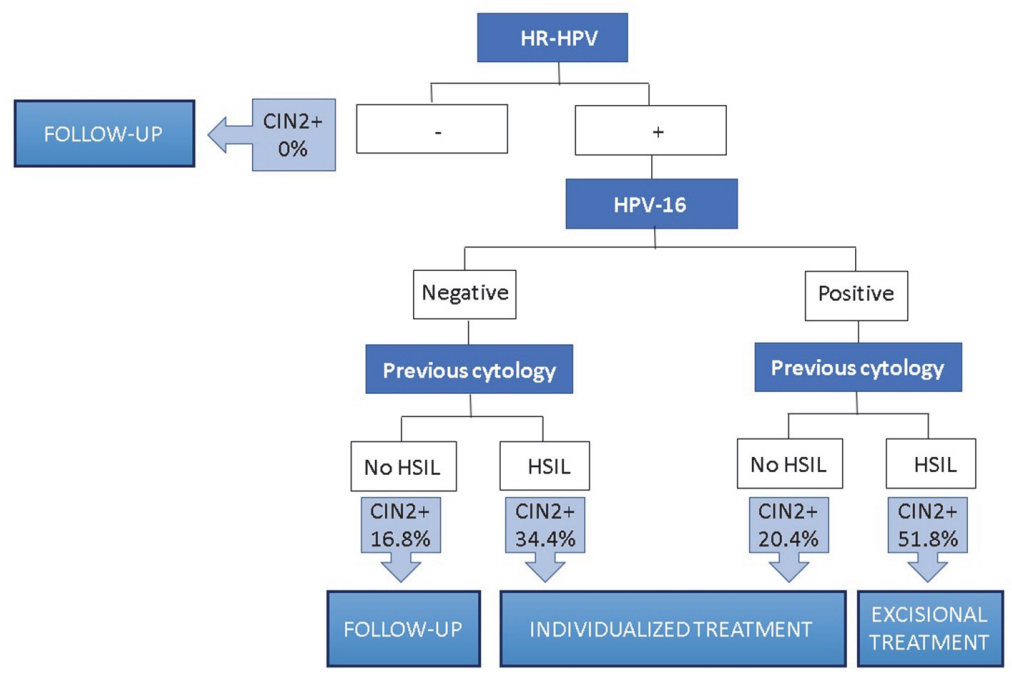

